\title{
Application of Symmetry Functions to Large Chemical Spaces Using Convolutional Neural Network
}

\author{
Balaranjan Selvaratnam, Ranjit T. Koodali, and Pere Miró
}

Department of Chemistry, University of South Dakota, 57069 Vermillion, SD, USA 


\section{Table of Contents}

$\begin{array}{lr}\text { Gaussian Parameters } & 4\end{array}$

$\begin{array}{ll}\text { Optimization of CNN Hyperparameters } & 5\end{array}$

Table S1. Parameters used for the angular symmetry functions. 3

Table S2. Results of hyperparameter optimization. 4

Table S3. Error for systems containing different number of elements. 5

Table S4. MAE error and $\mathrm{R}^{2}$ value of structures containing different elements. 6

Table S5. Element combinations and sample counts before and after the removal of low represented combinations. $\quad 8$

Figure S1. Loss curves for (a) QM9, (b) Materials dataset, (c) QM7-FF, and (d) QM7-DFT (inset $\begin{array}{ll}\text { shows the loss curve after } 50 \text { epochs). } & 10\end{array}$

Figure S2. Prediction of structures with 3 carbon member rings in the $\mathrm{C}_{7} \mathrm{H}_{10} \mathrm{O}_{2}$ isomer subset. 11

Figure S3. Prediction of structures with 4 carbon member rings in the $\mathrm{C}_{7} \mathrm{H}_{10} \mathrm{O}_{2}$ isomer subset. 12

Figure S4. Prediction of structures with 5 carbon member rings in the $\mathrm{C}_{7} \mathrm{H}_{10} \mathrm{O}_{2}$ isomer subset. 13

Figure S5. Prediction of structures with 6 carbon member rings in the $\mathrm{C}_{7} \mathrm{H}_{10} \mathrm{O}_{2}$ isomer subset. 14

Figure S6. Prediction of structures with 3 member rings and one of them is oxygen in the $\mathrm{C}_{7} \mathrm{H}_{10} \mathrm{O}_{2}$ isomer subset.

Figure S7. Prediction of structures with 4 member rings and one of them is oxygen in $\mathrm{C}_{7} \mathrm{H}_{10} \mathrm{O}_{2}$ isomer subset.

Figure S8. Prediction of structures with 5 member rings and one of them is oxygen in the $\mathrm{C}_{7} \mathrm{H}_{10} \mathrm{O}_{2}$ isomer subset.

Figure S9. Prediction of structures with 6 member rings and one of them is oxygen in the $\mathrm{C}_{7} \mathrm{H}_{10} \mathrm{O}_{2}$ isomer subset. 


\section{Gaussian Parameters}

For radial symmetry function 16 different $\eta$ were used and they are $0.0001,0.0002,0.0005,0.001$, $0.005,0.01,0.05,0.1,0.5,1,5,10,20,30,50$, and 100 . For angular symmetry function, the combinations of $\eta, \zeta$, and $\lambda$ are presented in Table $\mathrm{S} 1$.

Table S1. Parameters used for the angular symmetry functions.

\begin{tabular}{|c|c|c|c|}
\hline No & $\boldsymbol{\eta}$ & $\boldsymbol{\zeta}$ & $\boldsymbol{\lambda}$ \\
\hline 1 & 0.01 & 1 & 1 \\
\hline 2 & 0.01 & 1 & -1 \\
\hline 3 & 0.01 & 2 & 1 \\
\hline 4 & 0.01 & 2 & -1 \\
\hline 5 & 0.1 & 1 & 1 \\
\hline 6 & 0.1 & 1 & -1 \\
\hline 7 & 0.1 & 2 & -1 \\
\hline 8 & 0.1 & 2 & \\
\hline
\end{tabular}




\section{Optimization of CNN Hyperparameters}

Table S2. Results of hyperparameter optimization.

\begin{tabular}{|c|c|c|c|c|c|c|c|c|c|c|c|}
\hline \multirow[t]{2}{*}{ No } & \multicolumn{2}{|c|}{$\begin{array}{c}\text { Convolution } \\
\text { Kernel }\end{array}$} & \multicolumn{2}{|c|}{ Convolution Filters } & \multirow[t]{2}{*}{$\begin{array}{l}\text { Dense } \\
\text { Layers }\end{array}$} & \multirow[t]{2}{*}{$\begin{array}{l}\text { Batch } \\
\text { Size }\end{array}$} & \multirow{2}{*}{$\begin{array}{c}\text { Activation } \\
\text { Function } \\
\text { Conv/Dense }\end{array}$} & \multirow[t]{2}{*}{ Optimizer } & \multirow[t]{2}{*}{$\begin{array}{c}\text { Learning } \\
\text { Rate }\end{array}$} & \multirow[t]{2}{*}{ Loss } & \multirow[t]{2}{*}{$\begin{array}{c}\text { RMSE } \\
(\mathrm{kcal} / \mathrm{mol})\end{array}$} \\
\hline & Radial & Angular & Radial & Angular & & & & & & & \\
\hline 1 & $1 \mathrm{x} 1$ & $1 \times 1$ & 16,1 & 16,1 & 32,32 & 4 & ReLU & Adam & 0.001 & 130.37 & 11.42 \\
\hline 2 & $1 \times 1$ & $1 \times 1$ & 16,1 & 16,1 & 32,32 & 8 & ReLU & Adam & 0.001 & 132.97 & 11.53 \\
\hline 3 & $1 \times 1$ & $1 \times 1$ & 16,1 & 16,1 & 32,32 & 16 & ReLU & Adam & 0.001 & 120.67 & 10.98 \\
\hline 4 & $1 \times 1$ & $1 \times 1$ & 16,1 & 16,1 & 32,32 & 32 & ReLU & Adam & 0.001 & 175.88 & 13.26 \\
\hline 5 & $1 \times 1$ & $1 \times 1$ & 16,1 & 16,1 & 32,32 & 64 & ReLU & Adam & 0.001 & 238.03 & 15.43 \\
\hline 6 & $1 \times 1$ & $1 \times 1$ & 16,2 & 16,2 & 32,32 & 16 & ReLU & Adam & 0.001 & 124.81 & 11.17 \\
\hline 7 & $1 \times 1$ & $1 \times 1$ & 16,4 & 16,4 & 32,32 & 16 & ReLU & Adam & 0.001 & 84.33 & 9.18 \\
\hline 8 & $1 \times 1$ & $1 \mathrm{x} 1$ & 16,8 & 16,8 & 32,32 & 16 & ReLU & Adam & 0.001 & 108.14 & 10.40 \\
\hline 9 & $1 \times 1$ & $1 \mathrm{x} 1$ & $16,8,4$ & $16,8,4$ & 32,32 & 16 & ReLU & Adam & 0.001 & 107.02 & 10.34 \\
\hline 10 & $1 \times 1$ & $1 \times 1$ & $\begin{array}{c}16,12 \\
8,4 \\
\end{array}$ & $\begin{array}{c}16,12 \\
8,4\end{array}$ & 32,32 & 16 & ReLU & Adam & 0.001 & 143.34 & 11.97 \\
\hline 11 & $3 \times 3$ & $3 \times 3$ & 16,4 & 16,4 & 32,32 & 16 & ReLU & Adam & 0.001 & 138.71 & 11.78 \\
\hline 12 & $5 \times 5$ & $5 \times 5$ & 16,4 & 16,4 & 32,32 & 16 & ReLU & Adam & 0.001 & 160.82 & 12.68 \\
\hline 13 & $7 \times 7$ & $7 \times 7$ & 16,4 & 16,4 & 32,32 & 16 & ReLU & Adam & 0.001 & 135.86 & 11.65 \\
\hline 14 & $1 \times 1$ & $1 \times 1$ & 16,4 & 16,4 & 32 & 16 & ReLU & Adam & 0.001 & 116.38 & 10.79 \\
\hline 15 & $1 \times 1$ & $1 \times 1$ & 16,4 & 16,4 & 4,4 & 16 & ReLU & Adam & 0.001 & 204.32 & 14.29 \\
\hline 16 & $1 \times 1$ & $1 \mathrm{x} 1$ & 16,4 & 16,4 & 8,8 & 16 & ReLU & Adam & 0.001 & 189.39 & 13.76 \\
\hline 17 & $1 \times 1$ & $1 \times 1$ & 16,4 & 16,4 & 16,16 & 16 & ReLU & Adam & 0.001 & 301.34 & 17.36 \\
\hline 18 & $1 \times 1$ & $1 \times 1$ & 16,4 & 16,4 & 64,64 & 16 & ReLU & Adam & 0.001 & 147.90 & 12.16 \\
\hline 19 & $1 \mathrm{x} 1$ & $1 \mathrm{x} 1$ & 16,4 & 16,4 & 128,128 & 16 & ReLU & Adam & 0.001 & 122.37 & 11.06 \\
\hline 20 & $1 \mathrm{x} 1$ & $1 \mathrm{x} 1$ & 16,4 & 16,4 & 32,32 & 16 & Tanh & Adam & 0.001 & 548.49 & 23.42 \\
\hline
\end{tabular}


Table S3. Error for systems containing different number of elements.

\begin{tabular}{|c|c|c|}
\hline $\begin{array}{c}\text { Number of } \\
\text { Different Elements }\end{array}$ & $\begin{array}{c}\text { MAE } \\
\text { (meV/atom) }\end{array}$ & $\mathbf{R}^{\mathbf{2}}$ \\
\hline 1 & 418 & -4.86 \\
\hline 2 & 169 & 0.89 \\
\hline 3 & 106 & 0.95 \\
\hline 4 & 46 & 0.99 \\
\hline 5 & 37 & 0.99 \\
\hline 6 & 54 & 0.99 \\
\hline 7 & 61 & 0.95 \\
\hline
\end{tabular}


Table S4. MAE error and $\mathrm{R}^{2}$ value of structures containing different elements.

\begin{tabular}{|c|c|c|}
\hline Formula & $\begin{array}{c}\text { True Formation Energy } \\
\text { (meV/atom) }\end{array}$ & $\begin{array}{l}\text { Predicted Formation } \\
\text { Energy (meV/atom) }\end{array}$ \\
\hline $\mathrm{Ga}_{4}$ & 0.0 & -47.8 \\
\hline $\mathrm{Na}$ & 2.7 & -95.5 \\
\hline $\mathrm{Hf}$ & 181.1 & -451.1 \\
\hline $\mathrm{Sc}$ & 49.0 & -30.2 \\
\hline $\mathrm{Te}_{3}$ & 1.9 & 7.2 \\
\hline $\mathrm{Se}_{64}$ & 0.0 & -11.1 \\
\hline Th & 0.0 & 29.9 \\
\hline $\mathrm{P}_{2}$ & 92.4 & -68.5 \\
\hline $\mathrm{S}_{28}$ & 20.2 & -282.6 \\
\hline $\mathrm{Tl}_{4}$ & 20.8 & 27.5 \\
\hline $\mathrm{Ta}_{30}$ & 5.4 & 1497.5 \\
\hline $\mathrm{Hg}_{2}$ & 0.0 & 8.5 \\
\hline $\mathrm{As}_{2}$ & 0.0 & -8.0 \\
\hline Ho & 139.3 & -26.0 \\
\hline $\mathrm{Ni}$ & 0.0 & -6.4 \\
\hline $\mathrm{Sb}_{2}$ & 65.8 & -56.4 \\
\hline $\mathrm{C}_{2}$ & 3.7 & 397.6 \\
\hline $\mathrm{H}_{4}$ & 0.0 & -58.1 \\
\hline $\mathrm{C}_{10}$ & 145.2 & 747.9 \\
\hline $\mathrm{Bi}_{2}$ & 555.7 & 13.7 \\
\hline $\mathrm{Br}$ & 656.3 & 307.9 \\
\hline Y & 27.5 & 40.7 \\
\hline $\mathrm{F}_{4}$ & 0.0 & -2407.1 \\
\hline
\end{tabular}




\begin{tabular}{|c|c|c|}
\hline $\mathrm{S}$ & 1298.1 & 1795.5 \\
\hline $\mathrm{Pr}_{8}$ & 15.6 & 21.9 \\
\hline $\mathrm{F}_{4}$ & 0.0 & -2198.9 \\
\hline $\mathrm{Sc}_{6}$ & 130.0 & \begin{tabular}{|l|}
-32.4 \\
\end{tabular} \\
\hline $\mathrm{Sr}$ & 223.0 & -485.5 \\
\hline $\mathrm{C}_{2}$ & 4.4 & 394.3 \\
\hline $\mathrm{Ca}_{2}$ & 5.8 & -626.1 \\
\hline $\mathrm{S}_{48}$ & 1.3 & -253.6 \\
\hline $\mathrm{Rh}$ & 0.0 & 307.4 \\
\hline $\mathrm{Si}_{4}$ & 10.7 & 272.9 \\
\hline $\mathrm{Ce}_{4}$ & 52.5 & -15.3 \\
\hline $\mathrm{Ba}_{2}$ & 309.0 & -927.4 \\
\hline $\mathrm{Ti}$ & 63.4 & -26.1 \\
\hline $\mathrm{C}_{4}$ & 1299.4 & 488.8 \\
\hline $\mathrm{U}$ & 381.7 & 97.3 \\
\hline $\mathrm{S}_{56}$ & 38.0 & -224.7 \\
\hline $\mathrm{Ce}_{2}$ & 1.2 & 32.1 \\
\hline $\mathrm{Ta}_{30}$ & 4.9 & 1497.4 \\
\hline $\mathrm{S}_{18}$ & 438.1 & -897.6 \\
\hline $\mathrm{B}_{48}$ & 152.7 & 196.3 \\
\hline $\mathrm{Yb}_{2}$ & 5.4 & -136.9 \\
\hline $\mathrm{Np}_{4}$ & 80.7 & 42.4 \\
\hline $\mathrm{Ga}_{4}$ & 0.0 & $\begin{array}{l}-47.8 \\
\end{array}$ \\
\hline
\end{tabular}


Table S5. Element combinations and sample counts before and after the removal of low represented combinations.

\begin{tabular}{|c|c|c|c|}
\hline No & Elements & Counts (before) & Count (after) \\
\hline 1 & $\mathrm{C}, \mathrm{H}, \mathrm{N}, \mathrm{O}$ & 66,814 & 66,814 \\
\hline 2 & $\mathrm{C}, \mathrm{H}, \mathrm{O}$ & 45,770 & 45,770 \\
\hline 3 & $\mathrm{C}, \mathrm{H}, \mathrm{N}$ & 14,198 & 14,198 \\
\hline 4 & $\mathrm{C}, \mathrm{H}$ & 4,907 & 4,907 \\
\hline 5 & $\mathrm{C}, \mathrm{F}, \mathrm{H}, \mathrm{N}, \mathrm{O}$ & 1,062 & 1,062 \\
\hline 6 & $\mathrm{C}, \mathrm{F}, \mathrm{H}, \mathrm{N}$ & 736 & 736 \\
\hline 7 & $\mathrm{C}, \mathrm{F}, \mathrm{H}, \mathrm{O}$ & 250 & 250 \\
\hline 8 & $\mathrm{C}, \mathrm{F}, \mathrm{H}$ & 96 & 96 \\
\hline 9 & $\mathrm{C}, \mathrm{N}, \mathrm{O}$ & 26 & 14 \\
\hline 10 & $\mathrm{C}, \mathrm{F}, \mathrm{N}, \mathrm{O}$ & 14 & - \\
\hline 11 & $\mathrm{C}, \mathrm{N}$ & 4 & - \\
\hline 12 & $\mathrm{C}, \mathrm{F}, \mathrm{N}$ & 3 & - \\
\hline 13 & $\mathrm{C}, \mathrm{F}$ & 2 & - \\
\hline 14 & $\mathrm{H}, \mathrm{N}$ & 1 & - \\
\hline 15 & $\mathrm{H}, \mathrm{O}$ & 1 & 1 \\
\hline 16 & $\mathrm{~N}, \mathrm{O}$ & & \\
\hline
\end{tabular}



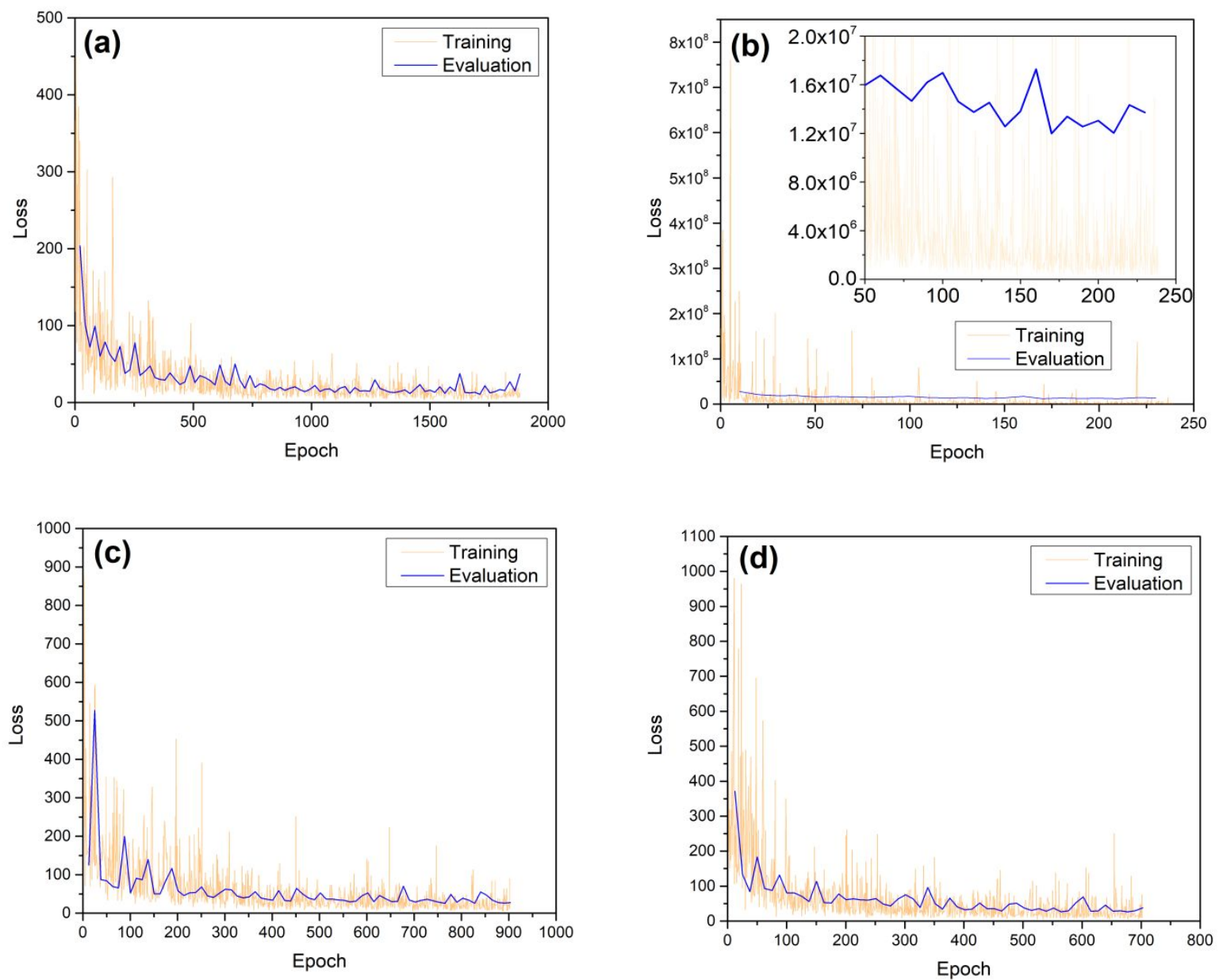

Figure S1. Loss curves for (a) QM9, (b) Materials dataset, (c) QM7-FF, and (d) QM7-DFT (inset shows the loss curve after 50 epochs). 


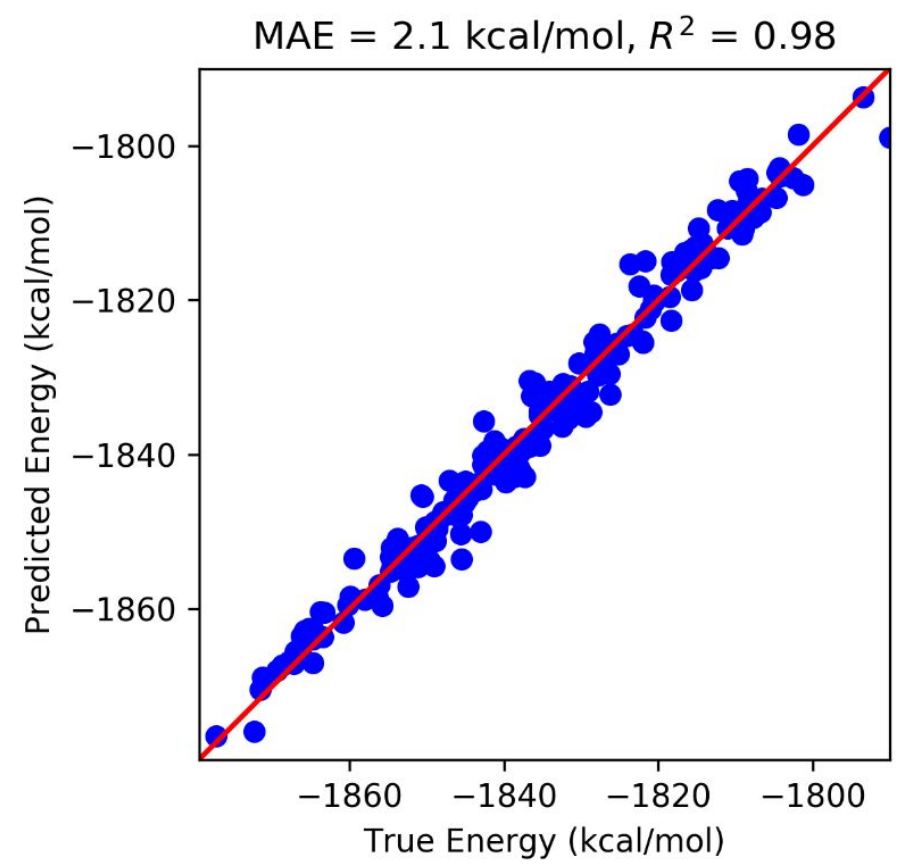

Figure S2. Prediction of structures with 3 carbon member rings in the $\mathrm{C}_{7} \mathrm{H}_{10} \mathrm{O}_{2}$ isomer subset. 


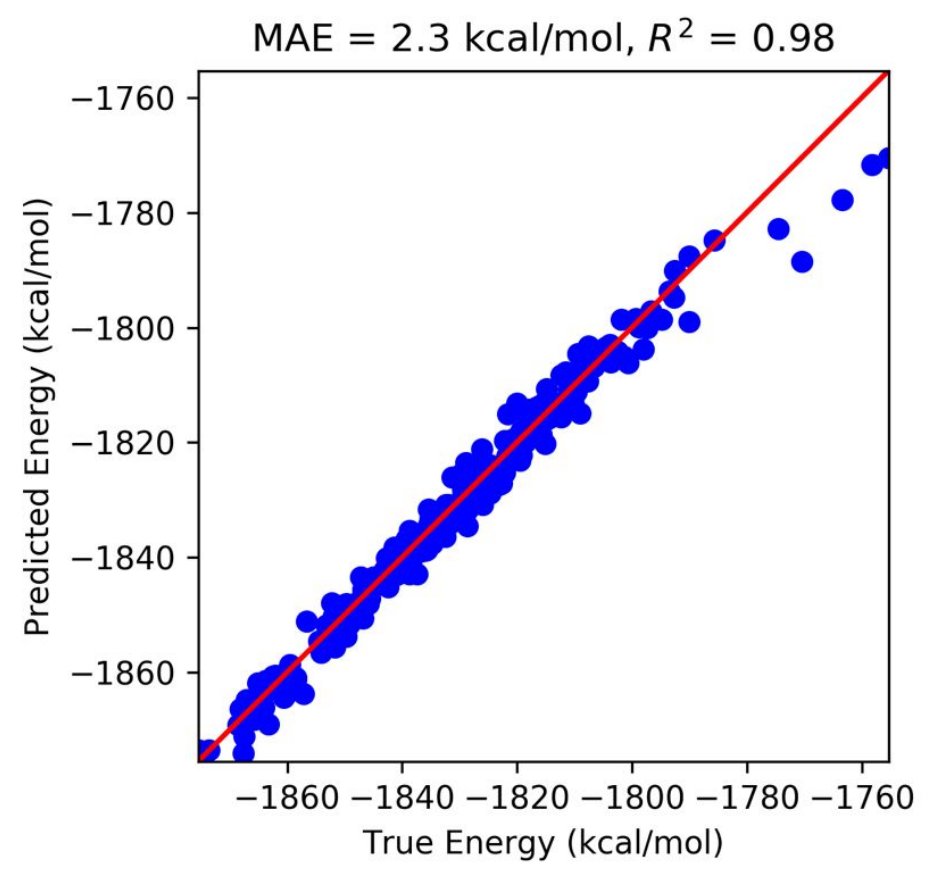

Figure S3. Prediction of structures with 4 carbon member rings in the $\mathrm{C}_{7} \mathrm{H}_{10} \mathrm{O}_{2}$ isomer subset. 


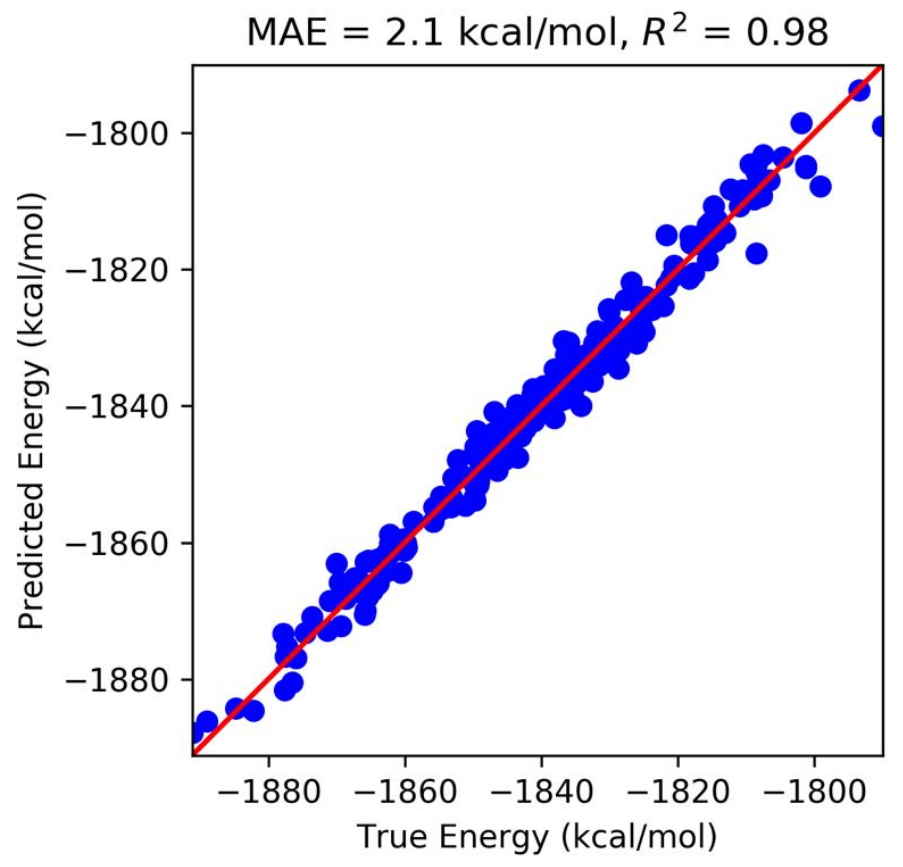

Figure S4. Prediction of structures with 5 carbon member rings in the $\mathrm{C}_{7} \mathrm{H}_{10} \mathrm{O}_{2}$ isomer subset. 


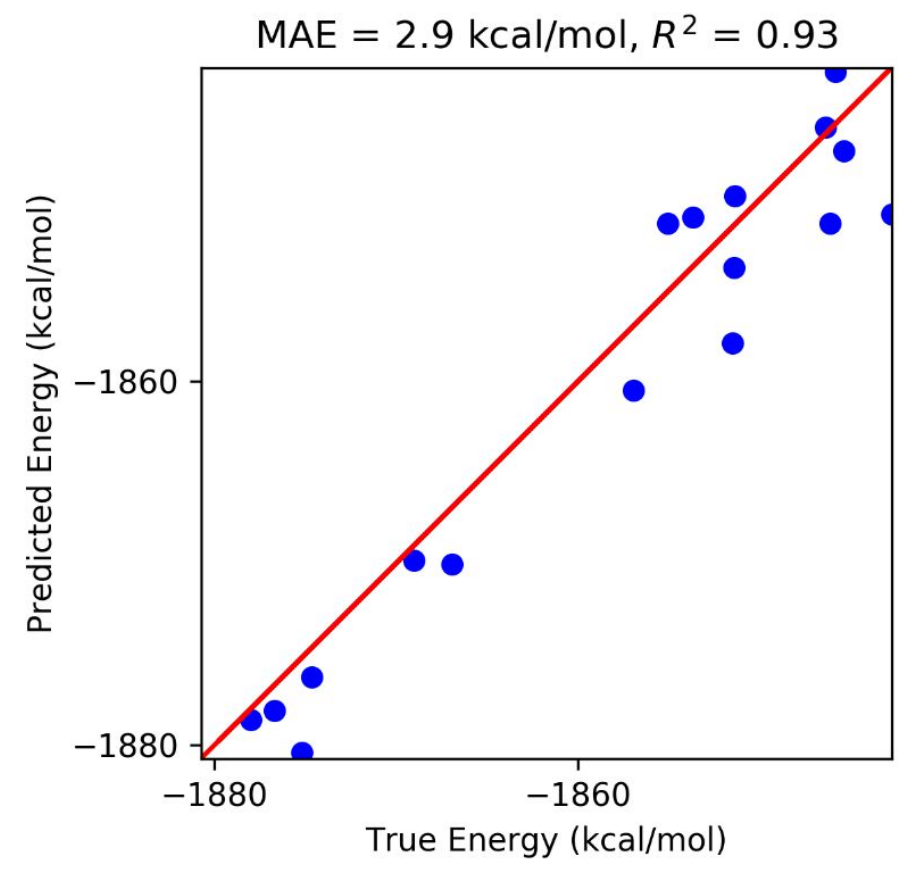

Figure S5. Prediction of structures with 6 carbon member rings in the $\mathrm{C}_{7} \mathrm{H}_{10} \mathrm{O}_{2}$ isomer subset. 


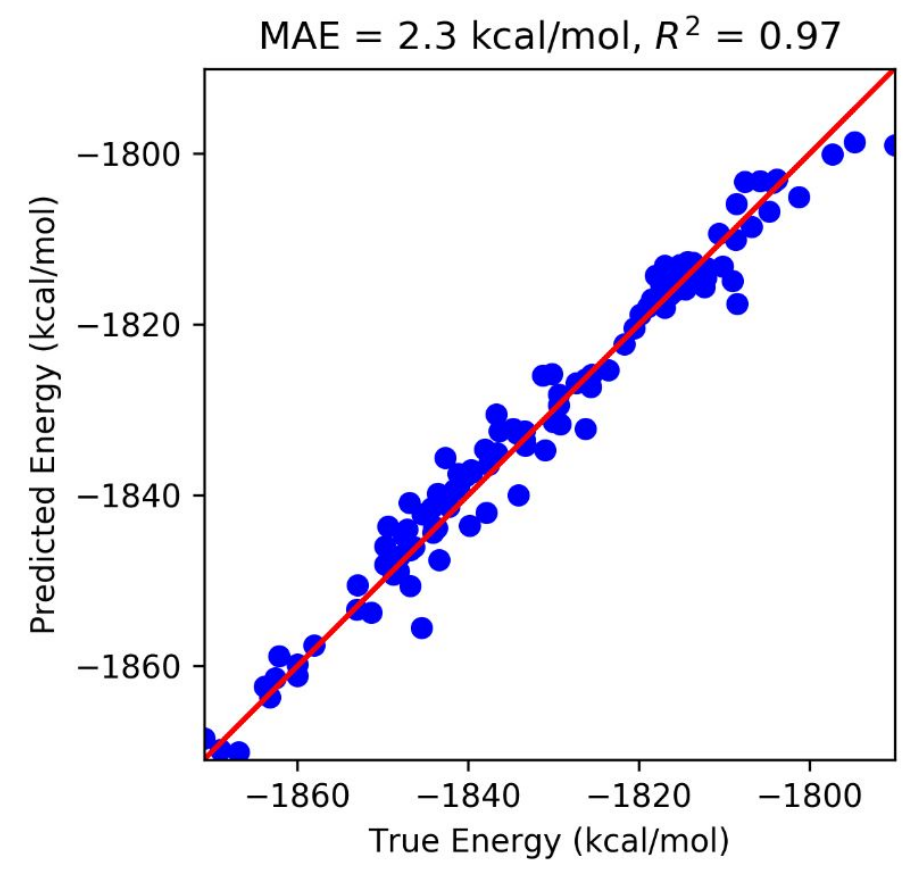

Figure S6. Prediction of structures with 3 member rings and one of them is oxygen in the $\mathrm{C}_{7} \mathrm{H}_{10} \mathrm{O}_{2}$ isomer subset. 


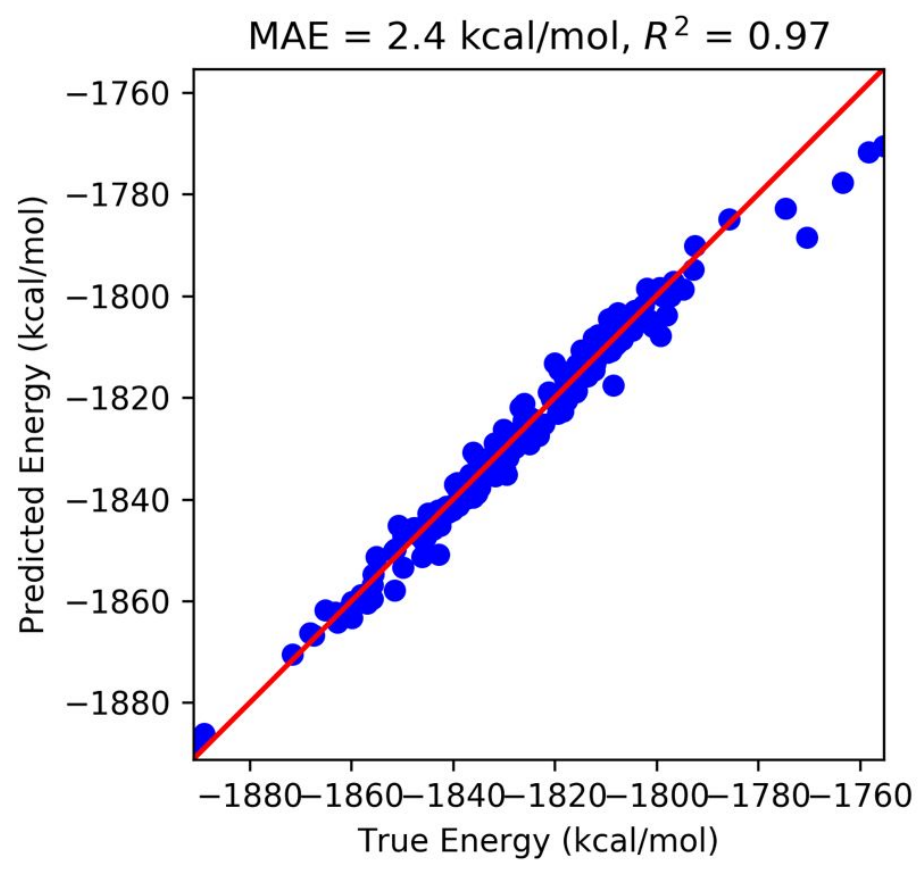

Figure S7. Prediction of structures with 4 member rings and one of them is oxygen in $\mathrm{C}_{7} \mathrm{H}_{10} \mathrm{O}_{2}$ isomer subset. 


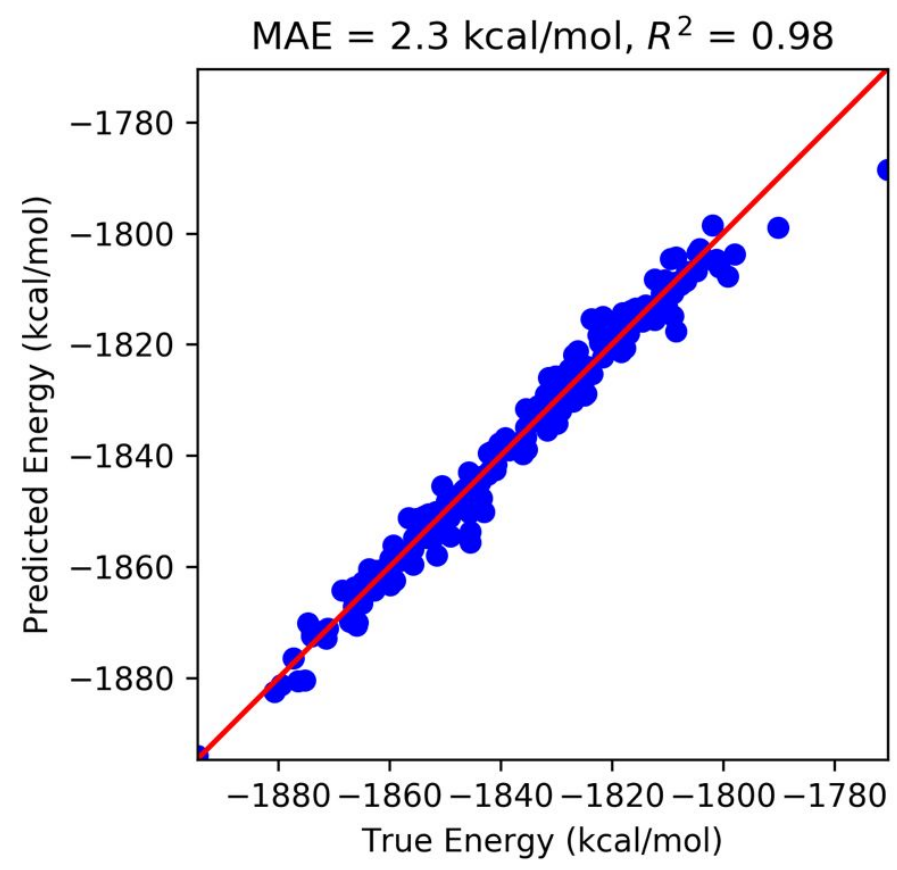

Figure S8. Prediction of structures with 5 member rings and one of them is oxygen in the $\mathrm{C}_{7} \mathrm{H}_{10} \mathrm{O}_{2}$ isomer subset. 


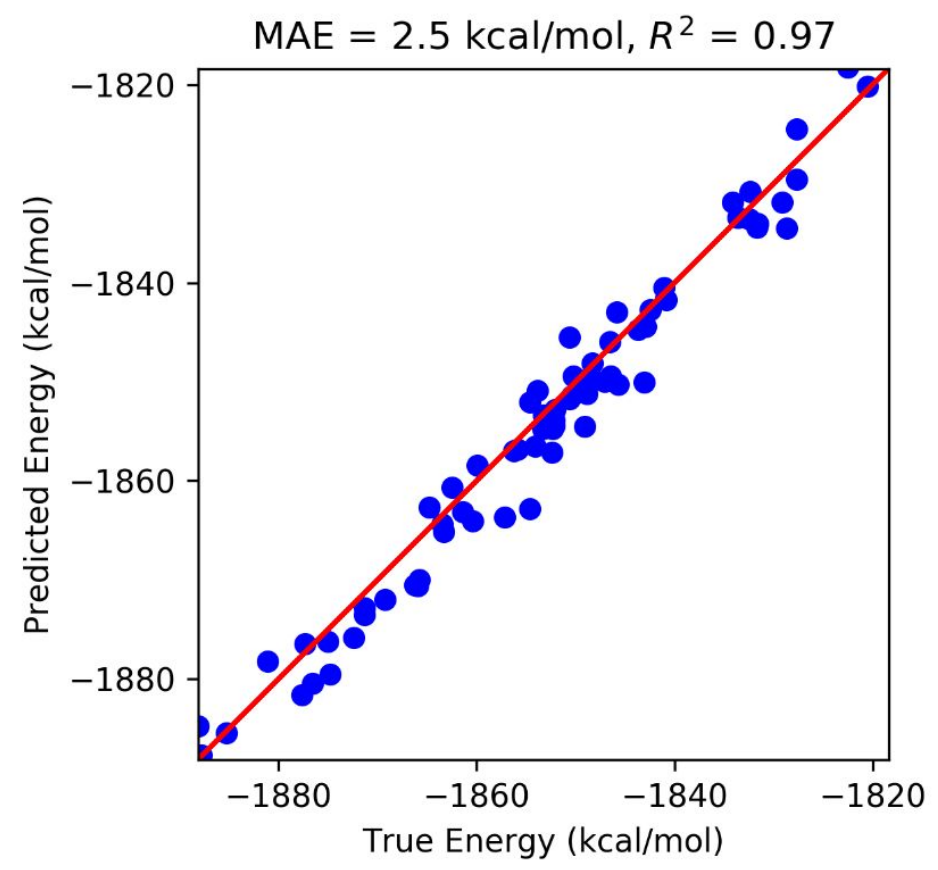

Figure S9. Prediction of structures with 6 member rings and one of them is oxygen in the $\mathrm{C}_{7} \mathrm{H}_{10} \mathrm{O}_{2}$ isomer subset. 